\title{
Sulfur fumigation processing of traditional Chinese medicinal herbs: beneficial or detrimental?
}

\author{
Winnie Lai Ting Kan, Bin Ma and Ge Lin* \\ School of Biomedical Sciences, The Chinese University of Hong Kong, Shatin, Hong Kong, China
}

Edited by:

De-An Guo, Shanghai Institute of Materia Medica, Chinese Academy of Sciences, China

Reviewed by:

Pierluigi Caboni, University of Cagliari, Italy

Shailendra Shivaji Gurav, Government College of Pharmacy, India

${ }^{*}$ Correspondence:

Ge Lin, School of Biomedical

Sciences, The Chinese University of Hong Kong, Basic Medical Sciences

Building, Shatin, New Territories,

Hong Kong, China.

e-mail: linge@cuhk.edu.hk
Majority of traditional Chinese medicine (TCM) herbs need to undergo post-harvesting processing to convert raw material into the form readily used for prescription. In general, processing procedures are either according to China Pharmacopeia or based on traditional methods. Recently sulfur fumigation is increasingly used to replace traditional sun-drying for its pesticidal and anti-bacterial properties in a cheap and convenient manner. However, to date information on effects of sulfur fumigation on herbal safety and efficacy are limited. This article addresses potential destructive effects of sulfur fumigation on herbal efficacy and safety through reviewing currently available information. Since recently increased numbers of studies have demonstrated that sulfur fumigation-induced dramatic changes in chemical profiles of various sulfur-fumigated herbs, consequent alteration of efficacy, and/or potential incidence of toxicity are suspected. Therefore comprehensive investigations on effects of sulfur fumigation on toxicity, chemical profiles, pharmacokinetics, and bioactivities of TCM herbs are timely to provide scientific basis for standardization and regulation of this currently common but potentially harmful processing method.

Keywords: sulfur fumigation, TCM herb processing, sulfur fumigation-induced chemical alteration, pharmacokinetic alteration, toxicity of sulfur dioxide, toxicity of sulfiting agents

\section{INTRODUCTION}

In the traditional Chinese medicine (TCM) practice, a personalized Chinese Materia Medica, usually in a mixed form, is prescribed to individual patients (Chan, 1995; Ye and He, 2010). The prescribed mixed form is called compound formula (Fufang) and commonly taken orally as an aqueous decoction. The compound formula consists of a complementary combination of various TCM materials, including medicinal herbs, animals, and minerals, which contain multiple bioactive compounds and interact synergistically with each other for enhanced efficacy at multiple targets (Tomlinson et al., 2000; Kan et al., 2008). Among Chinese Materia Medica used, TCM herbs are predominant. In China, the use of TCM remains the first-line treatment for many minor illnesses and chronic diseases. Recently, there is an increasing number of people worldwide who are using alternative medications especially TCM, and believe their therapeutic and safe values (Bent and Ko, 2004). For instance, it has been reported that up to $20 \%$ of cancer patients used herbal medicine to complement conventional chemotherapy regimens, enhance the immune system, improve general health, and reduce adverse effects from the conventional chemotherapy (Chiu et al., 2009; Damery et al., 2011). Studies also showed that $78 \%$ of patients admitted to hospital for acute cardiovascular diseases used natural health products, and of them $20 \%$ used herbal products and 9\% consumed TCM herbs (Alherbish et al., 2011).

Despite the surging popularity of TCM herbs, there are still many uncertainties surrounding its use. Often, not all of the bioactive and/or toxic constituents are identified in TCM herbs, so it complicates the process of delineating the mechanisms of beneficial action and adverse effects/toxicities, and therefore makes their quality control to be extremely difficult and challenging (Wang et al., 2009a). In general, medicinal herbs used in most Western countries are fresh or simply dried. Whereas, most of the TCM herbs have to be processed after harvesting by using physical and/or chemical methods to convert the raw materials to the readily used herbal forms called decoction pieces (Yinpian), which are then suitable for prescription or clinical usage (Zhao et al., 2010). Unfortunately, in addition to the numerous factors, such as herbal plant species, growing environment, harvesting time, storage condition, and contamination, which may significantly affect quality of TCM herbs (Tomlinson et al., 2000; Deng, 2002; Bent and Ko, 2004), unique and different post-harvesting processing methods, such as stir-frying, steaming, and calcining, for the same and different herbs, certainly cause more variations for the quality control of TCM herbs (Zhao et al., 2010). To make the situation even more complicated and problematic, some uncontrolled or poorly controlled processing procedures, such as the recently emerged sulfur fumigation, are often used by herbal farmers, producers, and manufactories in China. Recently, sulfur fumigation processing has attracted more attention due to its potential detrimental effect on the safety and efficacy of sulfur-fumigated TCM herbs. This article reviews the current situation and problems of sulfur fumigation of TCM herbs with emphasis on altercations of chemical profiles, pharmacokinetics, bioactivities, and even adverse effects/toxicities of TCM herbs caused by sulfur fumigation.

\section{CONVENTIONAL PROCESSING METHODS}

According to the principles of TCM, the main purpose of processing is to increase the efficacy and/or reduce the toxicity of TCM herbs. In addition, processing may be used to improve the odor or flavor of the herb, enhance the solubility of specific components 
in the herb, increase the purity by reduction of contaminants, and preserve the active ingredients (Zhao et al., 2010; Chang et al., 2011; Zhan et al., 2011). As early as 200 BC, TCM herbs were processed by burning and soaking in wine as documented in the Chinese “52 Bing Fang” (Prescriptions for 52 Diseases; Zhao et al., 2010). Currently 15 processing methods are recorded in Pharmacopeia of People's Republic of China (PRC; State Pharmacopoeia Committee, 2010). Some common processing methods, including slicing, steaming, boiling, stir-frying, calcining, and soaking in wine or vinegar, have been previously reported in few review articles, and thus are not described in details here (Chan, 1995; Bent and Ko, 2004; Wang et al., 2009a; Zhao et al., 2010).

One of the major post-harvesting factors affecting the efficacy and safety of TCM herbs are discrepancies in processing methods. Many studies demonstrated that various common processing methods drastically changed the chemical profile of TCM herbs. For instances, processing of Ligusticum Chuanxiong Rhizome (Chuanxiong, Ligusticum chuanxiong Hort., Umbelliferae) by sun-drying and stir-frying remarkably increased the contents of several bioactive ingredients, including senkyunolides I and $\mathrm{H}$, riligustilide, levistolide, and ferulic acid, but significantly reduced contents of three major constituents, senkyunolide A, $z$-ligustilide, and coniferyl ferulate in the herb via processing-induced hydroxylation, dimerization, and hydrolysis reactions (Li et al., 2007), although the former two major ingredients are also bioactive (Chan et al., 2007). Similarly, soaking Angelicae Sinensis Radix (Danggui, Angelica sinensis [Oliv.] Diels, Umbelliferae) in yellow wine increased and reduced quantities of ferulic acid and $z$-ligustilide, respectively (Zhan et al., 2011). Previously, several articles have reported and reviewed the general practice of the conventional processing methods recommended by Pharmacopeia of PRC and its beneficial effects of enhancing efficacy and reducing adverse effect/toxicity of TCM herbs via the alteration of chemical profiles of the herbs (Yu et al., 2005; Li et al., 2010a; Shaw, 2010; Zhao et al., 2010; Chang et al., 2011). Therefore, the details of these conventional processing methods are not described in this article.

\section{SULFUR FUMIGATION PROCESSING}

Traditionally, the roots and rhizomes of herbs were dried naturally under sun or in the shade, but in recent decades, this practice has been replaced by sulfur fumigation, a faster and cheaper method. Generally, herbs are placed in the upper levels of a closed chamber and sulfur powder is burned at the bottom of the chamber overnight. Sulfur dioxide is released into the chamber during this process and may penetrate into the herb (Wang et al., 2009b). Moreover, some herbal farmers even sprinkle sulfur powder on to the herbs to infiltrate sulfur into the herbs. Herbs are often treated by sulfur fumigation to decrease drying time, ward off insects, prevent molding and bacterial contamination, and give the herb a more pleasing white color (Upton, 2003; Wang et al., 2009b). Alternatively, herbs may be treated directly with sulfiting agents, such as sodium or potassium sulfite, and bisulfite or metabisulfite to protect the herb's moist appearance and maintain its color and freshness (Kim et al., 2000; Hayes et al., 2005). Although Pharmacopeia of PRC has prohibited sulfur fumigation for bleaching and processing all TCM herbs since 2005, there are no objectives of quantitative standards or well-defined regulations for acceptable levels of sulfur dioxide in herbs. Therefore, farmers continue to use this method to dry herbs with a higher profit margin and consumers are using the sulfur-fumigated herbs without awareness of their potential toxicity and possibly reduced or even no efficacy.

\section{TOXICITY ARISING FROM SULFUR FUMIGATION AND SULFITING AGENTS}

Exposure to sulfur dioxide seriously compromises human health. It has been reported that workers who performed sulfurization of apricots reported "asthma-like" symptoms such as itchy eyes, shortness of breath, cough, runny or stuffed nose, scratchy throat, and reduced pulmonary function when exposed to mean sulfur dioxide concentrations of $342 \mathrm{ppm}$ in a 1-h period (Koksal et al., 2003). In controlled human exposure studies, asthmatic subjects had increased airway resistance and decreased forced expiratory volume after being exposed to $400 \mathrm{ppb}$ sulfur dioxide for 5-10 min while exercising and showed cough, chest tightness, throat irritation, and other respiratory symptoms (Goodman et al., 2010). Sulfur dioxide forms sulfuric acid upon contacting with moist membranes and irritates the eyes, mucous membranes, and skin. Sulfuric acid also inhibits pulmonary particle clearance and induces mild bronchoconstriction, which is exacerbated in asthmatic patients (Komarnisky et al., 2003).

Sulfiting agents may lead to mild, moderate, and severe adverse events in the sulfite-sensitive asthmatic population (Lester, 1995), and specifically, dermatological symptoms (such as urticaria, angioedema, swelling), respiratory symptoms (such as dyspnea, wheezing, and bronchoconstriction), and gastrointestinal symptoms (such as nausea, vomiting, and diarrhea) have been clinically reported (Lester, 1995; Timbo et al., 2004; Vally et al., 2009). In more severe cases, sulfiting agents induced hypotension, cyanosis, shock, seizures, loss of consciousness, and even death (Yang and Purchase, 1985; Lester, 1995). Although the exact mechanism of sulfite-induced toxicity is unknown, it has been suggested that sulfite is a strong nucleophilic anion that reacts with immunological molecules (Gunnison and Jacobsen, 1987). Sulfite-sensitive asthmatics may have reduced levels and activity of sulfite oxidase, an enzyme mediating the oxidation of sulfite to sulfate, leading to higher susceptibility to sulfite intoxication (Yang and Purchase, 1985; Torun et al., 1989; Lester, 1995).

It has been reported that long-term inhalation of sulfur dioxide reduced lung function, increased oxidative stress, bronchial inflammation, and increased risk of lung cancer developed, and consumption of sulfur dioxide-containing herbs also caused clinical incidences of lung, liver, and kidney damage, blindness, skin rashes, asthma, and breathing difficulties (Nafstad et al., 2003; Rusconi et al., 2011). However, it is currently unknown whether these toxicities of sulfur-fumigated herbs originate only from the residual sulfur dioxide on the herb and/or from the chemical changes of the key compounds induced by sulfur fumigation in the herb. Although no extensive studies have been conducted on the safety of sulfur-fumigated herbs, it is suspected that long-term consumption of these herbs may be hazardous to health. Therefore, not only acceptable levels of sulfates or sulfites in the processed herbs need to be defined, the concentration of sulfur dioxide generated in and around the fumigation chamber also needs to be determined and governed to ensure the levels of sulfur dioxide, a common air 
pollutant, are at an acceptable level that will not induce harm to humans. In addition, understanding the impact of the chemical changes of the key ingredients in the herbs induced by sulfur fumigation on the safety of the sulfur-fumigated herbs is also timely and crucially important.

\section{CHEMICAL ALTERATION BY SULFUR FUMIGATION}

As summarized in Table 1, although limited information on the chemical changes induced by sulfur fumigation are available, the sulfur fumigation-induced alteration of chemical profiles of TCM herbs has been evidenced undoubtedly. Several studies investigated chemical changes of Paeoniae Radix Alba (Bai Shao, Paeonia lactiflora Pall., Paeoniaceae) after sulfur fumigation. In the sulfurfumigated Bai Shao, the amount of peoniflorin, a chemical marker for quality control of the herb, remarkably reduced, while a new compound peoniflorin sulfonate (Figure 1A) was found, which was further proved to be generated from the reaction of peoniflorin with sulfur dioxide in a mimic reaction even at room temperature (Wang et al., 2005). It has been demonstrated that almost $40 \%$ of peoniflorin was converted to peoniflorin sulfonate as early as $1 \mathrm{~h}$ after such reaction (Wang et al., 2005). Similarly, treating the herb with sodium bisulfite caused a reduction of peoniflorin content along with the formation of peoniflorin sulfonate (Hayes et al., 2005). Likewise, a reaction of pure peoniflorin with sodium bisulfite also yielded peoniflorin sulfonate (Hayes et al., 2005). In another study, two sulfonated components, namely peoniflorin sulfonate and benzoylpaeoniflorin sulfonate (Figure 1A), were formed in the sulfur fumigated-Bai Shao, while contents of the corresponding peoniflorin and benzoylpaeoniflorin were significantly decreased comparing with non-sulfur-fumigated herb (Cheng et al., 2010a).

The effect of sulfur fumigation on chemical profile of Angelicae Dahuricae Radix (Bai Zhi, Angelica dahurica [Fisch. ex Hoffm.] Benth. et Hook. f., Apiaceae) was also reported (Wang et al., 2009b). HPLC fingerprinting analyses were performed to analyze and compare chemical profiles of the sun-dried herb obtained from a cultivation base in China in operation under good agricultural practices (GAP) guidelines and from commercial sources that were confirmed to be sulfur-fumigated using sulfite residue testing. The results revealed that contents of the major furocoumarins were significantly reduced and at least $60 \%$ of imperatorin and almost all of oxypeucedanin was lost due to sulfur fumigation (Wang et al., 2009b). To further confirm these chemical changes, the herb was directly treated with sulfur dioxide in a mimic processing procedure. The results illustrated that contents of three major furocoumarins, namely imperatorin, isoimperatorin, and oxypeucedanin, were significantly decreased and converted to xanthotoxol, bergaptol, and oxypeucedanin hydrate, respectively (Figure 1B) were formed (Wang et al., 2009b).

A recent study on white ginseng (Shengshaishen), the processed Ginseng Radix et Rhizoma (Ren Shen, Panax ginseng C.A. Meyer, Araliaceae) also demonstrated the same processing problem. Some commercially available white ginseng samples, which should be processed by air-drying according to Pharmacopeia of PRC, were found to be also sulfur fumigated. A sensitive UPLC-Q-TOFMS/MS method used to analyze chemical profiles of both white ginseng and its decoction form (Du-Shen-Tang) revealed that contents of various ginsenosides were reduced and two ginsenoside sulfonate derivatives (Figure 1C) were found in both samples, however, the sulfonate substitution positions in these derivatives have not been definitively elucidated yet ( $\mathrm{Li}$ et al., 2010b).

The aforementioned studies and their findings provided evidence to reveal one of the key problems with sulfur fumigation. Sulfur fumigation may cause significant quantitative and qualitative changes of herbal bioactive ingredients, resulting in remarkable decrease or even disappearance of the bioactive compounds along with generation of new sulfonate derivatives. Consequently, not only pharmacokinetics and pharmacological activities of

Table 1 | Effects of sulfur fumigation or reaction with sulfiting agents on chemical constitution and pharmacokinetics of various TCM herbs.

\begin{tabular}{|c|c|c|c|c|}
\hline Herb & Processing & Chemical alteration & Pharmacokinetic alteration & Reference \\
\hline \multirow[t]{3}{*}{$\begin{array}{l}\text { Paeoniae Radix Alba } \\
\text { (Bai Shao) }\end{array}$} & Sulfur fumigation & $\begin{array}{l}\text { Formation of peoniflorin sulfonate } \\
\text { (9-16 mg/g herb) and } \\
\text { benzoylpaeoniflorin sulfonate } \\
(0.16-0.43 \mathrm{mg} / \mathrm{g} \text { herb) }\end{array}$ & $\begin{array}{l}\text { Oral absorption of peoniflorin sulfonate was } \\
\text { slower than that of peoniflorin. } \\
\text { Benzoylpaeoniflorin sulfonate but not } \\
\text { benzoylpaeoniflorin was present in blood } \\
\text { circulation after oral administration }\end{array}$ & Cheng et al. $(2010 a, b)$ \\
\hline & $\begin{array}{l}\text { Reaction with } \\
\text { sodium bisulfite }\end{array}$ & Formation of peoniflorin sulfonate & N.A. & Wang et al. (2005) \\
\hline & Sulfur fumigation & $\begin{array}{l}\text { Almost all of peoniflorin converted } \\
\text { to peoniflorin sulfonate }\end{array}$ & N.A. & Hayes et al. (2005) \\
\hline $\begin{array}{l}\text { Angelicae Dahuricae } \\
\text { Radix (Bai Zhi) }\end{array}$ & Sulfur fumigation & $\begin{array}{l}\text { Loss of major furocoumarins: i.e., at } \\
\text { least } 60 \% \text { loss of imperatorin, and } \\
\text { significant loss of isoimperatorin } \\
\text { and oxypeucedanin }\end{array}$ & N.A. & Wang et al. (2009b) \\
\hline $\begin{array}{l}\text { Ginseng Radix et } \\
\text { Rhizoma (Ren Shen) }\end{array}$ & Sulfur fumigation & $\begin{array}{l}\text { Formation of two ginsenoside } \\
\text { sulfonates }\end{array}$ & N.A. & Li et al. (2010b) \\
\hline
\end{tabular}

N.A., data not available. 


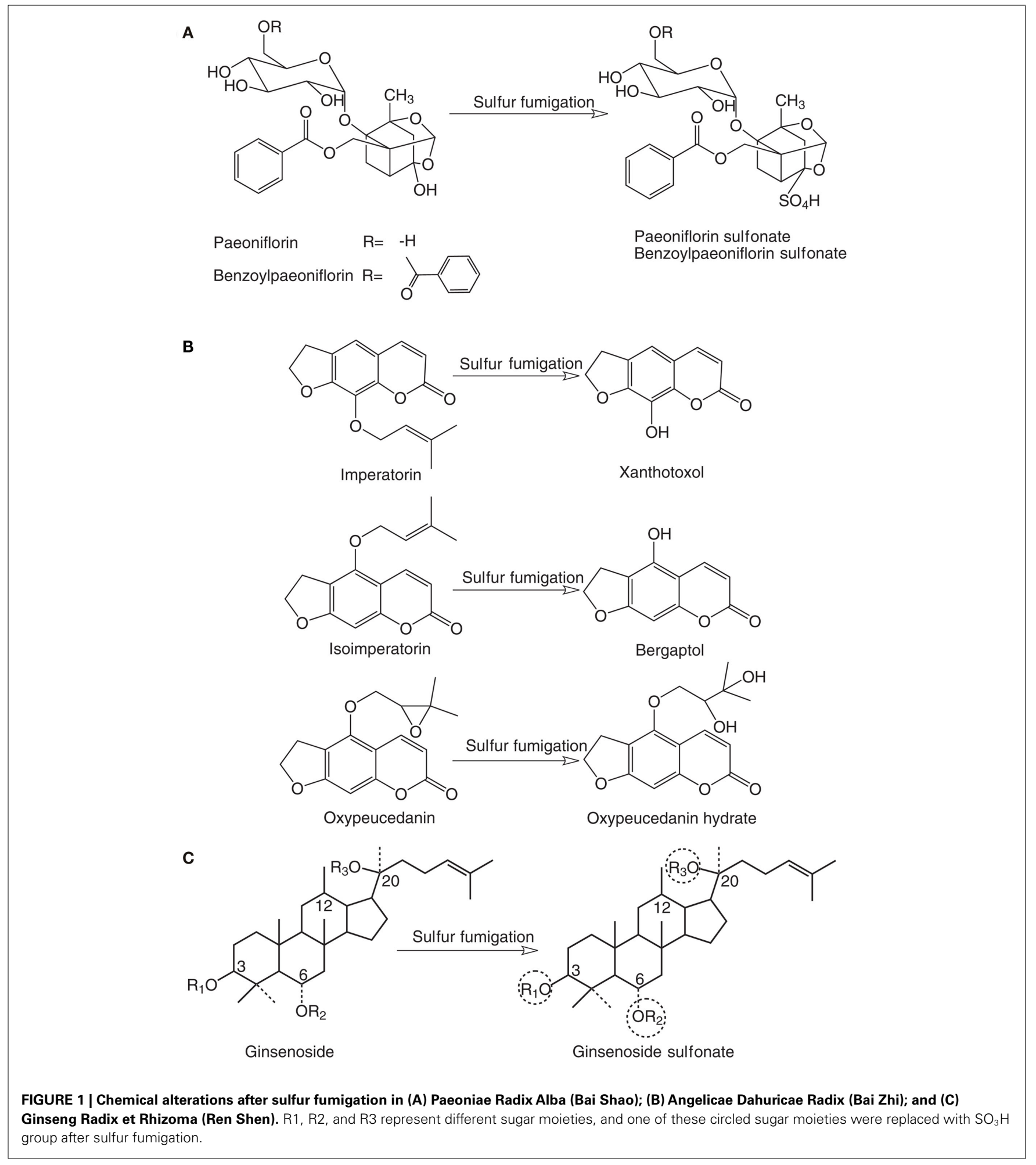

bioactive components are likely very different between sulfurfumigated herbs and conventionally processed herbs, changes in the undersigned bioactivities produced by sulfonate derivatives, which can be beneficial or harmful, may also occur inevitably in sulfur-fumigated herbs.

\section{PHARMACOKINETIC ALTERATION BY SULFUR FUMIGATION}

It is reasonable to hypothesize that the chemical profiling changes in the sulfur-fumigated herbs may result in pharmacokinetic alteration of herbal bioactive ingredients. However, to date, there is limited information in this regard and only one study 
examined pharmacokinetics of two main bioactive ingredients of Bai Shao, namely peoniflorin and benzoylpaeoniflorin, and also directly compared them with their sulfonate derivatives in mice via oral administration at $110 \mathrm{mg} / \mathrm{kg}$, although it did not compare pharmacokinetic fates of these ingredients after oral administration of sun-dried and sulfur-fumigated herbs (Cheng et al., 2010b). Compared with their parent compounds, both sulfonates had better metabolic stability as no metabolites of the sulfonates were found, which were suggested by the authors to be due to the replacement of hydroxyl group with sulfone group in the structures. The absence of the hydroxyl group prevented cleavage of the hemiketal-acetal system that normally occurs during metabolism to form peonimetabolins. For instance, owing to the better metabolic stability of the sulfonate derivative, peoniflorin had a significantly shorter halflife $\left(t_{1 / 2 \beta}: 112.3 \pm 48.36\right.$ vs $\left.247.1 \pm 65.35 \mathrm{~min}, p<0.05\right)$ than its sulfonate derivative. Moreover, it was unexpected that the oral absorbability of the sulfonate was significantly enhanced $\left(C_{\max }\right.$ : $5.01 \pm 2.21$ vs $4.36 \pm 1.13 \mu \mathrm{g} / \mathrm{ml}, p<0.05$ ) with a delayed absorption profile ( $T_{\max }: 30.0 \pm 0.0$ vs $56.0 \pm 8.9 \mathrm{~min}, p<0.05$ ) comparing with that of peoniflorin, and sulfonate derivative had a significantly higher oral bioavailability $\left(\mathrm{AUC}_{0-\infty}: 633.1 \pm 173.7\right.$ vs $519.1 \pm 155.6 \mu \mathrm{g} \cdot \mathrm{min} / \mathrm{ml}, p<0.05)$ than peoniflorin. Similarly, benzoylpaeoniflorin sulfonate significantly improved oral bioavailability ( $\mathrm{AUC}_{0-\infty}: 1486.7 \pm 499.5 \mu \mathrm{g} \cdot \mathrm{min} / \mathrm{ml}$ ), while benzoylpaeoniflorin was not absorbed because it was not detected in all plasma samples collected within $0-8.5 \mathrm{~h}$ after administration (Cheng et al., 2010b). However, whether such improvement of oral bioavailability and delay of absorption of sulfonate derivatives are common or unique in specific cases and whether systemic exposure of sulfonate derivatives affects herbal efficacy and/or toxicity are unknown and demands further systematic investigation.

\section{BIOACTIVITY ALTERATION BY SULFUR FUMIGATION}

It is also logical to suspect that the significant alteration of chemical profiles in sulfur-fumigated herbs will lead to significant changes in pharmacokinetic profiles of herbal bioactive components, and thus inevitably affect herbal efficacy and safety. However, to date only very limited information on the sulfur fumigation-induced changes of chemical and pharmacokinetic profiles are available, whereas, the impacts of sulfur fumigation on herbal pharmacological activities and adverse effects/toxicities due to the alteration of the chemical profiles have not been explored. Various researchers have expressed their views and concerns on the potential influences of sulfur fumigation on bioactivity and toxicity of TCM herbs. For instance, in the aforementioned study of Bai Zhi, based on the results of significant loss of the major active furocoumarins in sulfur-fumigated herb, the authors expected that herbal antiinflammation and anti-tumor activities, which were produced by furocoumarins, would be drastically reduced or even diminished (Okuyama et al., 1990; Ban et al., 2003). Nevertheless, no single published report has demonstrated the effects of chemical changes caused by sulfur fumigation on efficacy and safety of the processed herbs yet. Therefore, investigation in this regard is timely and warranted.

\section{CURRENT ISSUES WITH SULFUR FUMIGATION AND PERSPECTIVES}

Rigorous efforts have been made and are also continued to ensure good quality control in growth, harvesting, formulation, packaging, and marketing of TCM herbs and their compound formulae. However, information about standardized post-harvesting processing procedures is scarce due to the empirical and subjective nature of processing in its long history of practice. Although there is a general national standard for processing well-known TCM herbs, the standards differ among provinces and locations in China (Bent and Ko, 2004; Zhao et al., 2010). Often, processing is not considered as one of the major sources responsible for the lack of herbal efficacy and/or incidence of adverse effect/toxicity, and the public is unaware of how their consumed TCM herbs were processed and to what extent the quality of such herbs was affected (Shaw, 2010; Zhao et al., 2010). In recent years, particularly triggered by the prevalence of sulfur fumigation to process TCM herbs, increasing number of scientists are paying close attention to beneficial and detrimental effects of processing on the bioactivities of TCM herbs, and public consensus and media urge the need of implementing higher vigilance and tighter control of processing methods to increase safety, bioactivity, and credibility of TCM herbs (Deng, 2002; Bent and Ko, 2004; Shaw, 2010; Ye and He, 2010; Zhao et al., 2010). At the second Annual Meeting of the Specialty Committee of TCM Pharmaceutical Analysis of WFCMS \& International Conference on TCM Pharmaceutical Analysis (July 1st-3rd, 2011) in Chengdu, China, the potential effects of sulfur fumigation were addressed by several presentations, indicating the need for further studies in this area. Recently, China State Food and Drug Administration (SFDA) announced recommendations in that 11 TCM herbs, namely Achyranthis Bidentatae Radix (Niu Xi, Achyranthes bidentata Bl., Amaranthaceae), Asparagi Radix (Tian Dong, Asparagus cochinchinensis [Lour.] Merr., Liliaceae), Atractylodis Macrocephalae Rhizoma (Bai Zhu, Atractylodes macrocephala Koidz., Asteraceae), Bletillae Rhizoma (Bai Ji, Bletilla striata (Thunb.) Reichb. f., Orchidaceae), Codonopsis Radix (Dang Shen, Codonopsis pilosula (Franch.) Nannf., Campanulaceae), Dioscoreae Rhizoma (Shan Yao, Dioscorea opposita Thumb., Dioscoreaceae), Gastrodiae Rhizoma (Tian Ma, Gastrodia elata Bl., Orchidaceae), Kansui Radix (Gan Sui, Euphorbia kansui T.N. liou ex T.P. Wang, Euphorbiaceae), Paeoniae Radix Alba (Bai Shao, Paeonia lactiflora Pall., Paeoniaceae), Puerariae Thomsonii Radix (Fenge, Pueraria thomsonii Benth., Leguminosae), and Trichosanthis Radix (Tian Hua Fen, Trichosanthes kirilowii Maxim., Cucurbitaceae), are allowed to be processed by sulfur fumigation, but should have sulfur dioxide residual amount less than $400 \mathrm{ppm}(400 \mathrm{mg} / \mathrm{kg})$, while a residue limit of $150 \mathrm{ppm}(150 \mathrm{mg} / \mathrm{kg})$ is allowed for all other TCM herbs with prohibited sulfur fumigation (State Food and Drug Administration, 2011). However, scientific evidence supporting the rational for such residue limitations is unavailable. Currently, this recommendation is open for public opinion for future establishment of new regulations if public consensus is reached.

In addition to measuring sulfur dioxide residues, it is timely to develop suitable, convenient, and sensitive analytical methods for the determination of qualitative and quantitative changes in 
chemical components caused by sulfur fumigation, in order to assess (1) whether the herb has been sulfur fumigated; (2) which herbal bioactive components have changed after sulfur fumigation; (3) how the components have changed structurally; and (4) how much of the components have changed in terms of formation of new compounds and degradation of existing compounds. Furthermore, in vivo investigation of sulfur-fumigated herbs, including chemical and metabolite profiling, needs to be systematically conducted together with pharmacokinetic, bioactivity, and toxicity studies in parallel to acquire a better understanding of the effects of sulfur fumigation on efficacy and safety of TCM herbs. Only until the solid evidences have been obtained from the systematic and scientific studies, appropriate regulations governing which TCM herbs should not (processing-induced harm) or should

\section{REFERENCES}

Alherbish, A., Charrois, T. L., Ackman, M. L., Tsuyuki, R. T., and Ezekowitz, J. A. (2011). The prevalence of natural health product use in patients with acute cardiovascular disease. PLoS ONE 6, e19623. doi:10.1371/journal.pone.0019623

Ban, H. S., Lim, S. S., Suzuki, K., Jung, S. H., Lee, S., Lee, Y. S., Shin, K. H., and Ohuchi, K. (2003). Inhibitory effects of furanocoumarins isolated from the roots of Angelica dahurica on prostaglandin E2 production. Planta Med. 69, 408-412.

Bent, S., and Ko, R. (2004). Commonly used herbal medicines in the United States: a review. Am. J. Med. 116, 478-485.

Chan, K. (1995). Progress in traditional Chinese medicine. Trends Pharmacol. Sci. 16, 182-187.

Chan, S. S., Cheng, T. Y., and Lin, G. (2007). Relaxation effects of ligustilide and senkyunolide A, two main constituents of Ligusticum chuanxiong, in rat isolated aorta. J. Ethnopharmacol. 111, 677-680.

Chang, W. T., Choi, Y. H., Van der Heijden, R., Lee, M. S., Lin, M. K., Kong, H., Kim, H. K., Verpoorte, R., Hankemeier, T., Van der Greef, J., and Wang, M. (2011). Traditional processing strongly affects metabolite composition by hydrolysis in Rehmannia glutinosa roots. Chem. Pharm. Bull. 59, 546-552.

Cheng, Y. S., Peng, C., Zhang, H., and Liu, X. B. (2010a). Structural characterization of an artefact and simultaneous quantification of two monoterpenes and their artefacts of isolation in white-peony root. Helv. Chim. Acta 93, 565-572.

Cheng, Y., Peng, C., Wen, F., and Zhang, H. (2010b). Pharmacokinetic comparisons of typical constituents in white peony root and sulfur fumigated white peony root after oral administration to mice. J. Ethnopharmacol. 129, 167-173.

Chiu, J., Yau, T., and Epstein, R. J. (2009). Complications of traditional Chinese/herbal medicines (TCM) a guide for perplexed oncologists and other cancer caregivers. Support. Care Cancer 17, 231-240.

Damery, S., Gratus, C., Grieve, R., Warmington, S., Jones, J., Routledge, P., Greenfield, S., Dowswell, G., Sherriff, J., and Wilson, S. (2011). The use of herbal medicines by people with cancer: a cross-sectional survey. $\mathrm{Br}$. J. Cancer 104, 927-933.

Deng, J. F. (2002). Clinical and laboratory investigations in herbal poisonings. Toxicology 181-182, 571-576.

Goodman, J. E., Dodge, D. G., and Bailey, L. A. (2010). A framework for assessing causality and adverse effects in humans with a case study of sulfur dioxide. Regul. Toxicol. Pharmacol. 58, 308-322. (1987). Sulfite hypersensitivity: a critical review. CRC Crit. Rev. Toxicol. 17, 185-214.

Hayes, P. Y., Lehmann, R., Penman, K., Kitching, W., and De Voss, J. J. (2005). Sodium paeoniflorin sulfonate, a process derived artifact from paeoniflorin. Tetrahedron Lett. 46, 2615-2618.

Kan, W. L., Cho, C. H., Rudd, J. A., and Lin, G. (2008). Study of the antiproliferative effects and synergy of phthalides from Angelica sinensis on colon cancer cells. J. Ethnopharmacol. 120, 36-43.

Kim, Y. K., Koh, E., Park, S. Y., Chang, S. Y., Park, S. J., Na, W. I., and Kim, H. J. (2000). Determination of sulfite in oriental herbal medicines. J. AOAC Int. 83, 1149-1154.

Koksal, N., Hasanoglu, H. C., Gokirmak, M., Yildirim, Z., and Gultek, A. (2003). Apricot sulfurization: an occupation that induces
Gunnison, A. F., and Jacobsen, D. W.

(processing-induced benefit or no change) be processed by sulfur fumigation with well-controlled procedures can be established.

Nevertheless, the aforementioned quality control of postharvesting processing is one of many crucial steps, such as GAP on herbal farms, good manufacturing practice (GMP) in herbal manufacturing, and good warehousing practice (GWP) for storage and distribution, for the assurance of good quality of TCM herbs. This task is extremely challenging and needs tremendous efforts from close collaborations among various parties including government authorities, regulatory agencies, TCM farmers, pharmaceutical industry, consumers, and scientists. Such collaborative work will boost local and international credibility of TCM herbs, and ultimately result in the production and sale of safer TCM herbs with higher efficacy for public health.

an asthma-like syndrome in agricultural environments. Am. J. Ind. Med. 43, 447-453.

Komarnisky, L. A., Christopherson, R. J., and Basu, T. K. (2003). Sulfur: its clinical and toxicologic aspects. Nutrition 19, 54-61.

Lester, M. R. (1995). Sulfite sensitivity: significance in human health. J. Am. Coll. Nutr. 14, 229-232.

Li, S. L., Song, J. Z., Qiao, C. F., Zhou, Y., Qian, K., Lee, K. H., and Xu, H. X. (2010a). A novel strategy to rapidly explore potential chemical markers for the discrimination between raw and processed Radix Rehmanniae by UHPLC-TOFMSN with multivariate statistical analysis. J. Pharm. Biomed. Anal. 51, 812-823.

Li, S. L., Lai, S. F., Song, J. Z., Qiao, C. F., Liu, X., Zhou, Y., Cai, H., Cai, B. C., and Xu, H. X. (2010b). Decoctinginduced chemical transformations and global quality of Du-ShenTang, the decoction of ginseng evaluated by UPLC-Q-TOF-MS/MS based chemical profiling approach. J. Pharm. Biomed. Anal. 53, 946-957.

Li, S. L., Yan, R., Tam, Y. K., and Lin, G. (2007). Post-harvest alteration of the main chemical ingredients in Ligusticum chuanxiong Hort. (Rhizoma Chuanxiong). Chem. Pharm. Bull. 55, 140-144.

Nafstad, P., Haheim, L. L., Oftedal, B., Gram, F., Holme, I., Hjermann, I., and Leren, P. (2003). Lung cancer and air pollution: a 27 year follow up of 16209 Norwegian men. Thorax 58, 1071-1076.

Okuyama, T., Takata, M., Nishino, H., Nishino, A., Takayasu, J., and Iwashima, A. (1990). Studies on the antitumor-promoting activity of naturally occurring substances. II. Inhibition of tumor-promoterenhanced phospholipid metabolism by umbelliferous materials. Chem. Pharm. Bull. 38, 1084-1086.
Rusconi, F., Catelan, D., Accetta, G., Peluso, M., Pistelli, R., Barbone, F., Di Felice, E., Munnia, A., Murgia, P., Paladini, L., Serci, A., and Biggeri, A. (2011). Asthma symptoms, lung function, and markers of oxidative stress and inflammation in children exposed to oil refinery pollution. $J$. Asthma 48, 84-90.

Shaw, D. (2010). Toxicological risks of Chinese herbs. Planta Med. 76, 2012-2018.

State Food and Drug Administration. (2011). Regulations on the Residue Limit of Sulfur Dioxide in the TCMs and Their Formulations. Available at: http://www.sfda.gov.cn/WS01/CL00 51/63039.html [Accessed June 13, 2011].

State Pharmacopoeia Committee. (2010). Pharmacopoeia of the People's Republic of China, 2010 Edn. Beijing: China Medical Science and Technology Press.

Timbo, B., Koehler, K. M., Wolyniak, C., and Klontz, K. C. (2004). Sulfites - a food and drug administration review of recalls and reported adverse events. J. Food Prot. 67, 1806-1811.

Tomlinson, B., Chan, T. Y., Chan, J. C., Critchley, J. A., and But, P. P. (2000). Toxicity of complementary therapies: an eastern perspective. J. Clin. Pharmacol. 40, 451-456.

Torun, M., Bayhan, A., and Yentur, G. (1989). Response of allergic and normal persons to sulfiting agents in wine: determination of thiosulfate excretion in urine. Clin. Chem. 35, 1792-1793.

Upton, R. (2003). American Herbal Pharmacopoeia and Therapeutic Compendium: Dang Gui Root Angelica sinensis (Oliv.) Diels (Scotts Valley, CA: CRC Press), 1-40.

Vally, H., Misso, N. L., and Madan, V. (2009). Clinical effects of sulfite additives. Clin. Exp. Allergy 39, 1643-1651. 
Wang, J., van der Heijden, R., Spruit, S., Hankermeier, T., Chan, K., van der Greef, J., Xu, G., and Wang, M. (2009a). Quality and safety of Chinese herbal medicines guided by a systems biology perspective. J. Ethnopharmacol. 126, 31-41.

Wang, X. H., Xie, P. S., Lam, C. W., Yan, Y. Z., and Yu, Q. X. (2009b). Study of the destructive effect to inherent quality of Angelicae dahuricae radix (Baizhi) by sulfur-fumigated process using chromatographic fingerprinting analysis. J. Pharm. Biomed. Anal. 49, 1221-1225.

Wang, Q., Liu, R., Guo, H., Ye, M., Huo, C., Bi, K., and Guo, D. (2005). Simultaneous LC determination of major constituents in red and white peony root. Chromatographia 62, 581-588.

Yang, W. H., and Purchase, E. C. (1985). Adverse reactions to sulfites. CMAJ 133, 865-867, 880.

Ye, X. F., and He, J. (2010). The bright future of Chinese herbal medicine: only after a twisty road. Contemp. Clin. Trials 31, 508-509.

Yu, L., Sun, S. Q., Fan, K. F., Zhou, Q., and Noda, I. (2005). Research on processing medicinal herbs with multi-steps infrared macro-fingerprinting method. Spectrochim. Acta A Mol. Biomol. Spectrosc. 62, 22-29.

Zhan, J. Y., Zheng, K. Y., Zhu, K. Y., Bi, C. W., Zhang, W. L., Du, C. Y., Fu, Q., Dong, T. T., Choi, R. C.,
Tsim, K. W., and Lau, D. T. (2011). Chemical and biological assessment of Angelicae Sinensis Radix after processing with wine: an orthogonal array design to reveal the optimized conditions. J. Agric. Food Chem. 59, 6091-6098.

Zhao, Z., Liang, Z., Chan, K., Lu, G., Lee, E. L., Chen, H., and Li, L. (2010). A unique issue in the standardization of Chinese materia medica: processing. Planta Med. 76, 1975-1986.

Conflict of Interest Statement: The authors declare that the research was conducted in the absence of any commercial or financial relationships that could be construed as a potential conflict of interest.
Received: 14 July 2011; accepted: 07 December 2011; published online: 27 December 2011.

Citation: Kan WLT, Ma B and Lin G (2011) Sulfur fumigation processing of traditional Chinese medicinal herbs: beneficial or detrimental? Front. Pharmacol. 2:84. doi: 10.3389/fphar.2011.00084

This article was submitted to Frontiers in Ethnopharmacology, a specialty of Frontiers in Pharmacology.

Copyright ( $\odot 2011$ Kan, Ma and Lin. This is an open-access article distributed under the terms of the Creative Commons Attribution Non Commercial License, which permits non-commercial use, distribution, and reproduction in other forums, provided the original authors and source are credited. 